\title{
GLOBAL STABILITY PROPERTIES OF A HYPERBOLIC SYSTEM ARISING IN PATTERN FORMATION $\dagger$
}

\author{
CARLOS MARTEL and JOSÉ M. VEGAł
}

\author{
E.T.S.I. Aeronáuticos, Universidad Politécnica de Madrid, 28040 Madrid, Spain
}

Key words and phrases: Hyperbolic system, global stability, sub- and super-solutions, oscillatory instability, pattern formation.

\section{INTRODUCTION}

This paper deals with the following hyperbolic system

$$
\begin{aligned}
& u_{t}-u_{x}=u(\lambda-u-\alpha v), \\
& v_{t}+v_{x}=v(\lambda-v-\alpha u),
\end{aligned}
$$

in $-1<x<1$ and $t \geq 0$, with boundary conditions

and initial conditions

$$
\begin{array}{ll}
v=R u & \text { at } x=-1, \\
u=R v & \text { at } x=1,
\end{array}
$$

$$
u=u_{0}(x)>0, \quad v=v_{0}(x)>0 \quad \text { at } t=0,
$$

where $u_{0}$ and $v_{0}$ are $\mathfrak{e}^{1}$-functions satisfying (1.3), (1.4) and the additional compatibility conditions

$$
\begin{array}{ll}
v_{0}^{\prime}+R u_{0}^{\prime}=(\alpha-1)(R-1) R u_{0}^{2} & \text { at } x=-1, \\
u_{0}^{\prime}+R v_{0}^{\prime}=(\alpha-1)(1-R) R v_{0}^{2} & \text { at } x=1 .
\end{array}
$$

Hete, the real parameter $\lambda$ is a bifurcation parameter, while $R>0$ and $\alpha$ are real constants.

The model (1.1)-(1.5) is a sub-model of the normal form that is obtained in the weaklynonlinear analysis of the so-called oscillatory instability (see, e.g. [1]). That instability is of interest in many physical problems and has been observed experimentally in, e.g. binary fluid convection [2-5], isothermal double diffusive convection [6], pure Rayleigh-Bénard convection (as a secondary instability of rolls) [7-9], the Taylor-Couette system [10, 11], several thermocapillary flows [12-14], electrodynamic convection in nematic liquid crystals [15-18], and several combustion systems [19-21]. 
Let us now explain briefly how these equations and boundary conditions are obtained. We consider a system of PDEs of the form

$$
\frac{\partial u}{\partial T}=G\left(\frac{\partial^{2}}{\partial X^{2}}, u, \varepsilon\right) \quad \text { in }-L<X<L, \quad T \geq 0,
$$

where $u=\left(u_{1}, \ldots, u_{N}\right)$ is a function of the space variable $X$ and the time $T, \varepsilon$ is a control parameter, and $G$ is a nonlinear differential operator (invariant under space translations, $X \rightarrow X+c$ and reflection, $X \rightarrow-X)$ such that $G\left(\partial^{2} / \partial X^{2}, 0, \varepsilon\right) \equiv 0$ (the uniform state $u \equiv 0$ satisfies the system of PDEs for all $\varepsilon$ ). Also, we assume that the length $L$ is large (as compared with the wavelength $k_{0}^{-1}$ defined below) and impose appropriate boundary conditions at $x= \pm L / 2$, also invariant under reflection, and initial conditions at $T=0$, such that the resulting mathematical problem is well-posed.

Let $\varphi\left(\omega, k^{2}, \varepsilon\right)=0(\omega=$ growth rate, $k=$ wavenumber) be the (complex) dispersion relation of the linearized version of $(1.8)$ about $u=0$. We assume that the growth rates of the most unstable modes are of the form

$$
\begin{aligned}
& \omega= \pm i \Omega+c_{ \pm} \varepsilon \pm i d\left(k-k_{0}\right)-e_{ \pm}\left(k-k_{0}\right)^{2}+o(|\varepsilon|)+o\left(\left|k-k_{0}\right|^{2}\right), \\
& \omega= \pm i \Omega+c_{ \pm} \varepsilon \mp i d\left(k+k_{0}\right)-e_{ \pm}\left(k+k_{0}\right)^{2}+o(|\varepsilon|)+o\left(\left|k+k_{0}\right|^{2}\right),
\end{aligned}
$$

as $\varepsilon \rightarrow 0$ and $k \rightarrow \pm k_{0}$, while $\operatorname{Re} \omega<0$ otherwise (see Fig. 1). Here the constants $\Omega, k_{0}$ and $d$ are real, while $c_{ \pm}$and $e_{ \pm}$are complex and

$$
\begin{gathered}
\Omega>0, \quad k_{0}>0, \quad d \neq 0, \quad \operatorname{Re} c_{ \pm}>0, \quad \operatorname{Re} e_{ \pm}>0, \\
c_{-}=\bar{c}_{+}, \quad e_{-}=\bar{e}_{+},
\end{gathered}
$$

where $i$ is the imaginary unit, and Re and overbars stand for the real part and the complex conjugate respectively. Notice that the associated neutrally stable modes at $\varepsilon=0$ are of the form

$$
u=U_{0}\left[A \exp \left(i \Omega T+i k_{0} X\right)+B \exp \left(i \Omega T-i k_{0} X\right)\right]+\text { c.c. }
$$

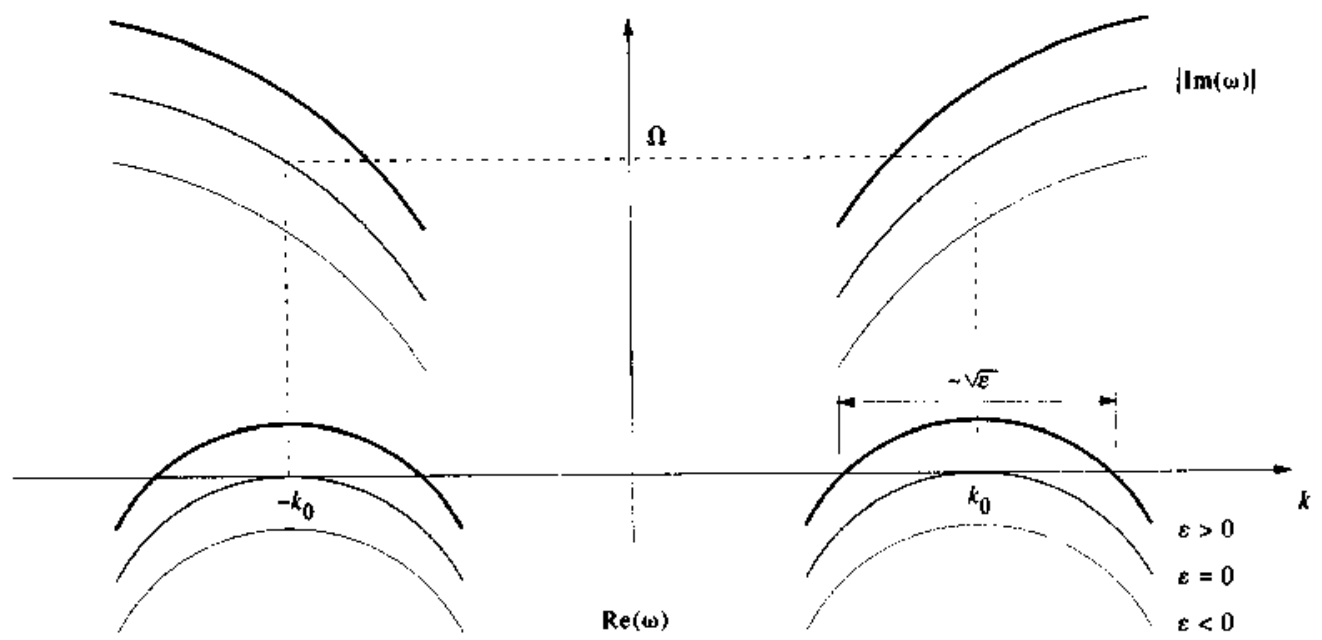

Fig. 1. Dispersion relation near criticality. 
for a certain eigenvector $U_{0} \in \mathbb{C}^{N}$, where the complex constants $A$ and $B$ are arbitrary and c.c. stands for the complex conjugate. That mode is the superposition of two counter-propagating wavetrains. The weakly nonlinear evolution of that pair of waves, as $\varepsilon \rightarrow 0$, is described by allowing the small complex amplitudes $A$ and $B$ to depend weakly on $X$ and $T$ and considering higher order terms (proportional to $A^{2}, B^{2},|A|^{2},|B|^{2}, A_{X}, B_{X}, A_{X X}, B_{X X}, A_{T}, B_{T}, A|A|^{2}$, $\left.A|B|^{2}, B|A|^{2}, B|B|^{2}, \ldots\right)$ in (1.8). If, in addition, the appropriate solvability conditions are applied at each asymptotic order, then the following equations are obtained for the evolution of $A$ and $B$,

$$
\begin{aligned}
& A_{T}=e_{+} A_{X X}+d A_{X}+c_{+} \varepsilon A+g_{1} A|A|^{2}+g_{2} A|B|^{2}+\cdots, \\
& B_{T}=e_{+} B_{X X}-d B_{X}+c_{+} \varepsilon B+g_{1} B|B|^{2}+g_{2} B|A|^{2}+\cdots,
\end{aligned}
$$

where the coefficients $c_{+}, d$ and $e_{+}$are as defined above, and the complex coefficients $g_{1}$ and $g_{2}$ depend on the quadratic and cubic nonlinear terms of (1.8). Equations (1.12) and (1.13) may be obtained quite directly by symmetry considerations (see [22]) but the actual values of the coefficients $g_{1}$ and $g_{2}$ must be obtained by the process described above (which usually leads to quite tedious calculations). Equations (1.12) and (1.13) apply only in the bulk, i.e. as $-L<X<L$ and $|X \pm L|>1$. In addition we must consider two boundary layers near the ends of the interval, $0<X+L \sim 1$ and $0<L-X \sim 1$. When the weakly nonlinear behavior in these boundary layers is calculated and matching conditions with the solution in the bulk are applied, the following boundary conditions are obtained for the complex amplitudes $A$ and $B$,

$$
\begin{array}{cc}
B=r A+\cdots \quad \text { at } X=-L, \quad A=r B+\cdots & \text { at } X=L, \\
d\left(B_{X}+r A_{X}\right)=\left(g_{1}-g_{2}\right) r\left(|r|^{2}-1\right) A|A|^{2}+\cdots & \text { at } X=-L, \\
d\left(A_{X}+r B_{X}\right)=\left(g_{2}-g_{1}\right) r\left(|r|^{2}-1\right) B|B|^{2}+\cdots & \text { at } X=L,
\end{array}
$$

where the constants $d, g_{1}$ and $g_{2}$ are as defined above, and the complex constant $r \neq 0$ is a reflection coefficient that depends on the linearized version of (1.8) and on the associated boundary conditions at $X= \pm L$. Conditions (1.14) account for linear reflection of the wavetrains at the boundary layers, that absorb a part of the incoming wave if $|r|<1$, and amplify it if $|r|>1$; they were first obtained by Cross [23]. The nonlinear boundary conditions (1.15) and (1.16) take into account higher order effects, and have been obtained by the authors of this paper $[24,25]$.

Several remarks about equations (1.12)-(1.13) and boundary conditions (1.14)-(1.16) are in order:

(a) For the sake of clarity we considered the 1-D problem (1.8), but (1.12)-(1.16) are also obtained from multidimensional problems, see $[24,25]$.

(b) The normal form (1.12)-(1.16) depends on the PDEs (1.8) and on the associated boundary conditions only through the values of its coefficients, that may be calculated by the process described above. Alternatively, the coefficients may be estimated by a direct quantitative comparison between the solutions of (1.12)-(1.16) and either experimental results from the underlying physical problem or direct numerical simulations on (1.8).

(c) Equations (1.12)-(1.16) are invariant under the transformation $A \rightarrow B, B \rightarrow A$ and $X \rightarrow-X$ [as a consequence of the invariance of $(1.8)$ and the associated boundary conditions under spatial reflection]. 
(d) The coefficient of $A_{X}$ and $-B_{X}$ in (1.12)-(1.13), $d$ (that is, the group velocity), is nonzero [see (1.9)]; in fact, the generic assumption $d \neq 0$ is essential in the derivation of the boundary conditions (1.15)-(1.16). On the other hand, the weakly nonlinear level of the asymptotic analysis requires essentialiy that

$$
\left|A_{X X}\right| \&\left|A_{X}\right| \ll|A| \ll 1, \quad\left|B_{X X}\right| \ll\left|B_{X}\right| \ll|B| \ll 1,
$$

and this implies that equations (1.12) and (1.13) necessarily contain terms that are not of the same order of magnitude. This could be seen as a strong difficulty [26] but, although it leads to some subtleties in the analysis, it allows us to consider two distinguished simpler submodels of (1.12)-(1.16) that are more amenable to purely analytical treatment. For an asymptotic derivation of these submodels, see $[24,25]$; here we only give a brief description of them.

The trivial steady state of (1.12)-(1.16), $A=B=0$, corresponds to the basic steady state of (1.8), $u=0$. After linearizing (1.12)-(1.16) about that steady state, it is readily seen that it is asymptotically stable only if

$$
\varepsilon<\varepsilon_{c}=-\left(d /\left(2 L \operatorname{Re} c_{+}\right)\right) \log |r|+o\left(L^{-l}\right)
$$

and two distinguished limits must be considered

$$
\left|\varepsilon-\varepsilon_{c}\right| \sim L^{-2}, \quad|A| \sim|B| \sim L^{-1},
$$

and

$$
|\varepsilon|-L^{-1}, \quad|A| \sim|B|-L^{-1 / 2}
$$

In the limit (1.17) we must consider one spatial scale, $x=X / L \sim 1$ and two time scales, $t=T / L \sim 1$ and $\tau=T / L^{2} \sim 1$, and the evolution of the complex amplitudes, $A$ and $B$, in the slower time scale, $\tau \sim 1$, is given by a nonlocal, complex Ginzburg-Landau equation [24, 25]. Particular versions of this nonlocal submodel, applying only to the case of perfectly reflecting boundaries, $|r|=1$, were obtained independently in $[27,28]$ by means of formal, two-timing perturbation methods; for a rigorous justification of the derivation, see [29]. That equation predicts some interesting complex large-time behavior of the wavetrains [24, 25], but it applies only in a very narrow region close to the instability limit, $\left|\varepsilon-\varepsilon_{c}\right| \sim L^{-2}$ [see (1.17)].

The limit (1.18) corresponds to a wider interval for the bifurcation parameter, $\left|\varepsilon-\varepsilon_{c}\right| \sim L^{-1}$. If we re-scale the variables and the bifurcation parameters as

$$
\varepsilon=\mu / L, \quad A=A_{1} / L^{1 / 2}, \quad B=B_{1} / L^{1 / 2}, \quad X=x L, \quad T=t L /|d|
$$

with $|\mu| \sim\left|A_{1}\right| \sim\left|B_{1}\right| \sim t \sim 1$, then (1.12)-(1.16) may be written in first approximation as

$$
\begin{gathered}
A_{1 t}=\left(d A_{1 x}+\mu c_{+} A_{1}+g_{1} A_{1}\left|A_{1}\right|^{2}+g_{2} A_{1}\left|B_{1}\right|^{2}\right) /|d|, \\
B_{1 t}=\left(-d B_{1 x}+\mu c_{+} B_{1}+g_{1} B_{1}\left|B_{1}\right|^{2}+g_{2} B_{1}\left|A_{1}\right|^{2}\right) /|d|, \\
B_{1}=r A_{1} \quad \text { at } x=-1, \quad A_{1}=r B_{1} \quad \text { at } x=1, \\
d\left(B_{1 x}+r A_{1 x}\right)=\left(g_{1}-g_{2}\right) r\left(|r|^{2}-1\right) A_{1}\left|A_{1}\right|^{2} \quad \text { at } x=-1, \\
d\left(A_{1 x}+r B_{1 x}\right)=\left(g_{2}-g_{1}\right) r\left(|r|^{2}-1\right) B_{1}\left|B_{1}\right|^{2} \quad \text { at } x=1,
\end{gathered}
$$


where, in particular, we have ignored the second derivatives (that is, diffusion and dispersion) in (1.19)-(1.20). But a natural question arises on whether this approximation makes sense. This is a stability question (the second derivatives may be ignored if small perturbations depending on intermediate spatial scales, $\xi=X / \delta \sim 1$, with $1 \leqslant \delta \ll L$, are damped out as time proceeds) that has been partially solved in $[24,25]$, where it has been shown that the second derivatives cannot be ignored if either

$$
\operatorname{Re} g_{1}>0, \quad \text { or } \operatorname{Re} g_{1}<0 \quad \text { and } \quad \operatorname{Re}\left(e_{+} \bar{g}_{1}\right)>0 .
$$

while they can be neglected if

$$
\operatorname{Re} g_{1}<0 \quad \text { and } \quad 2 \operatorname{Re}\left(e_{+}\right) \operatorname{Re}\left(g_{1}\right)<\operatorname{Re}\left(e_{+} \bar{g}_{1}\right)<0 \text {. }
$$

Notice that these conditions depend only on $g_{1}$ and $c_{+}$(but neither on the remaining parameters, nor on initial conditions). Some partial analytical results (concerning particular values of the remaining parameters) and some numerical simulations with (1.12)-(1.16) made us to conjecture that the approximation is also valid if $\operatorname{Re} g_{1}<0$ and $\operatorname{Re}\left(e_{+} \vec{g}_{t}\right) \leq$ $2 \operatorname{Re}\left(c_{+}\right) \operatorname{Re}\left(g_{1}\right)$. Anyway, if (1.25) holds then the submodel (1.19)-(1.23) applies. Then, if the group velocity $d$ is positive, and the new variables and parameters

$$
\begin{gathered}
u=-2 \operatorname{Re} g_{1}\left|A_{1}\right|^{2} /|d|, \quad v=-2 \operatorname{Reg} g_{1}\left|B_{1}\right|^{2} /|d|, \\
\lambda=2 \mu \operatorname{Re} c_{+} /|d|, \quad \alpha=\operatorname{Re} g_{2} / \operatorname{Reg} g_{1}, \quad R=|r|^{2},
\end{gathered}
$$

are introduced, then $(1.19)-(1.21)$ is rewritten as (1.1)-(1.4), while (1.22)-(1.23) is rewritten as

$$
\begin{array}{ll}
v_{x}+R u_{x}=(\alpha-1)(R-1) R u^{2} & \text { at } x=-1, \\
u_{x}+R v_{x}=(1-\alpha)(R-1) R v^{2} & \text { at } x=1 .
\end{array}
$$

If the group velocity $d$ is negative, then (1.1)-(1.4), (1.26)-(1.27) is still obtained if

$$
u=-2 \operatorname{Re} g_{1}\left|B_{1}\right|^{2} /|d|, \quad v=-2 \operatorname{Re} g_{1}\left|A_{1}\right|^{2} /|d|, \quad R=|r|^{-2},
$$

while $\lambda$ and $\alpha$ are defined as above.

Notice that, since $\operatorname{Re} g_{1}<0$ [see (1.25)], then $u$ and $v$ are nonnegative. In fact, we shall assume that

$$
u>0 \text { and } \quad v>0 \quad \text { in }-1 \leq x \leq 1, \quad t=0,
$$

and this will imply that $u>0$ and $v>0$ if $-1 \leq x \leq 1, t>0$. Also, the additional conditions (1.26)-(1.27) are precisely compatibility conditions for the hyperbolic problem (1.1)-(1.4) such that, if they hold at $t=0$ [as is assumed, see (1.6)-(1.7)] then they hold for all $t>0$, and allow the unique solution of (1.1)-(1.4) to be a $\mathfrak{C}^{1}$-solution.

The dynamics of (1.1)-(1.4) is in good qualitative and quantitative agreement with experimental results in the literature concerning physical systems exhibiting the oscillatory instability, see $[24,30]$. The dynamics is quite rich if $\alpha>1$, and includes at least period-doubling sequences, intermittency, quasiperiodic behavior and crises associated with symmetry gaining $[24,30]$. If $\alpha<1$ instead, numerical results seem to show that the dynamics of (1.1)-(1.4) is very simple; namely, the solutions either converge to a steady state or blow up. The main object 

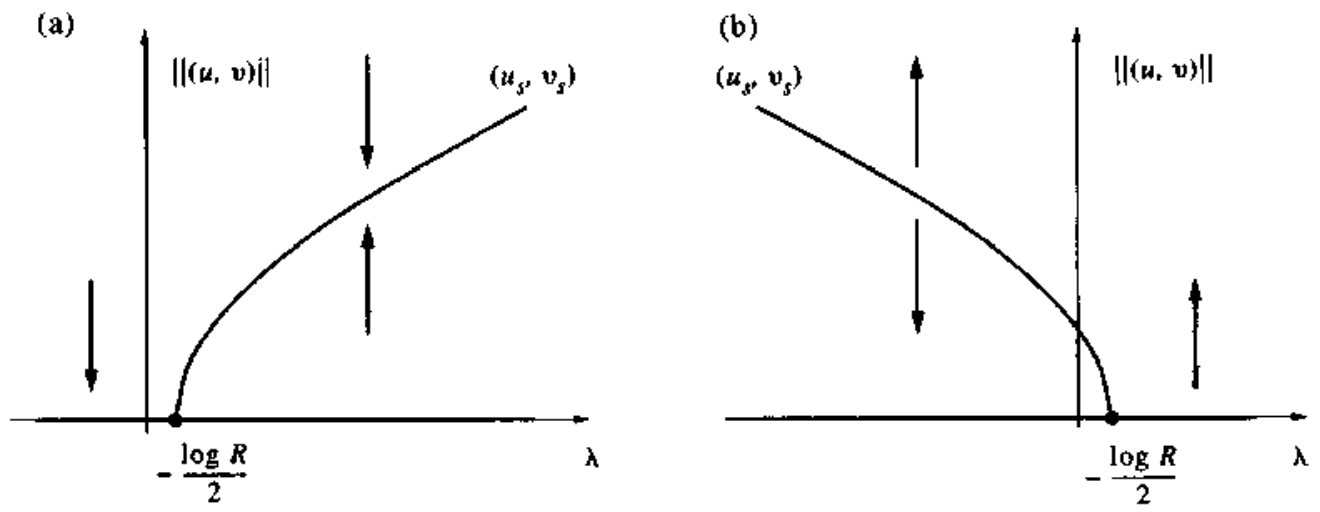

Fig. 2. Sketch of the large time behavior of the solutions of (1.1)-(1.4) for: (a)-1 $<\alpha<1$ and (b) $\alpha<-1$.

of this paper is to show analytically that this conjecture is true, namely we shall show that (see Fig. 2 for a sketch of these results):

(a) If $\alpha \leq-1$ then (1.1)-(1.7) possess solutions that diverge, in finite time if $\alpha<-1$ or as $t \rightarrow \infty$ if $\alpha=-1$, while if $\alpha>-1$, then the solutions of (1.1)-(1.7) are uniformly bounded in $0 \leq t<\infty$.

(b) If $\alpha>-1$ and $\lambda<-(\log R) / 2$, then every solution of (1.1)-(1.7) converges uniformly to $u=v=0$ as $t \rightarrow \infty$.

(c) If $-1<\alpha<1$ and $\lambda>-(\log R) / 2$ then as $t \rightarrow \infty$ every solution of (1.1)-(1.7) converges uniformly to a unique steady state, $\left(u_{s}, v_{s}\right)$, such that $u_{s}>0$ and $v_{s}>0$ in $-1 \leq x \leq 1$.

More precisely, the following results concerning the problem (1.1)-(1.7) will be proven:

(i) That problem has a unique solution in $0 \leq t<T$, for some $T \leq \infty$ (Theorem 3.1).

(ii) If $\alpha<-1, \lambda<-(\log R) / 2$ and the initial conditions are sufficiently large, then the solution becomes unbounded in finite time (Theorem 3.8B); if the initial conditions are sufficiently small, then the solution converges uniformly to $(u, v)=(0,0)$ as $t \rightarrow \infty$ (Remark 3.9).

(iii) If $\alpha<-1$ and $\lambda \geq-(\log R) / 2$ then every solution becomes unbounded in finite time (Theorem 3.8A).

(iv) If $\alpha=-1$ and $\lambda=-(\log R) / 2$ then (1.1)-(1.3) has infinitely many steady states (Section 2).

(v) If $\alpha=-1$ and $\lambda>-(\log R) / 2$ then every solution becomes unbounded as $t \rightarrow T$, where $T \leq \infty$ (Theorem 3.8A).

(vi) If $\alpha>-1$ and $\lambda \leq-(\log R) / 2$ then every solution converge uniformly to $(u, v)=(0,0)$ as $t \rightarrow \infty$ (Theorems 3.7 and 3.11 ).

(vii) If $-1<\alpha<1$ and $\lambda>-(\log R) / 2$ then, as $t \rightarrow \infty$, every solution converge uniformly to a steady state, $\left(u_{s}, v_{s}\right)$, such that $u_{s}>0$ and $v_{s}>0$ in $-1 \leq x \leq 1$ (Theorems 3.5 and 3.14).

(viii) If $\alpha \geq 1$ and $\lambda>-(\log R) / 2$ then every solution is globally defined and uniformly bounded in $0 \leq t<\infty$ (Theorem 3.11).

The paper is organized as follows. The steady states of (1.1)-(1.4) are briefly considered in Section 2. Global existence and uniqueness, along with properties (b) and (c) above are considered in Section 3. Finally, some concluding remarks are drawn in Section 4. 


\section{STEADY STATES}

The steady states of (1.1)-(1.4) are given by

$$
\begin{aligned}
& \mathrm{d} u / \mathrm{d} x=-u(\lambda-u-\alpha v), \\
& \mathrm{d} v / \mathrm{d} x=v(\lambda-v-\alpha u),
\end{aligned}
$$

in $-1<x<1$, with boundary conditions

$$
v=R u \quad \text { at } x=-1, \quad u=R v \quad \text { at } x=1 .
$$

As pointed out in Section 1, we are interested only in those solutions of (2.1)-(2.3) such that $u \geq 0$ and $v \geq 0$ in $-1 \leq x \leq 1$; then uniqueness of the ODEs (2.1)-(2.2) and the boundary conditions (2.3) readily imply that either

$$
u>0 \text { and } v>0 \text { in }-1 \leq x \leq 1
$$

or $u=v=0$ in $-1 \leq x \leq 1$.

The solutions of (2.1)-(2.4) may be found in closed form if $\alpha=-1,0$ and 1 . If $\alpha=-1$ and $\lambda=-(\log R) / 2$, then $(2.1)-(2.4)$ has the one-parameter family of solutions

$$
u(x)=v(-x)=\left(\frac{\log R}{2}\right) R^{x / 2} /\left[c-R^{x / 2}-R^{-x / 2}\right],
$$

if $R \neq 1$, where the parameter $c$ varies in the interval $-\infty<c<2$ if $R<1$, and in the interval $\sqrt{R}+1 / \sqrt{R}<c<\infty$ if $R>1$; if $R=1$, then

$$
u(x)=v(x)=c,
$$

with the parameter $c$ such that $0<c<\infty$. If $\alpha=-1$ and $\lambda \neq-(\log R) / 2$, then $(2.1)-(2.4)$ has no solution.

If $\alpha=0$ and $\lambda>-(\log R) / 2$, then (2.1)-(2.4) has a unique solution, that is given by

$$
u(x)=v(-x)=\lambda\left(R \mathrm{e}^{\lambda}-\mathrm{e}^{-\lambda}\right) /\left[R \mathrm{e}^{\lambda}-\mathrm{e}^{-\lambda}-(R-1) \mathrm{e}^{\lambda x}\right]
$$

if $\lambda \neq 0$, while if $\lambda=0$ then

$$
u(x)=v(-x)=(R-1) /[R+1-x(R-1)] .
$$

If $\alpha=0$ and $\lambda \leq-(\log R) / 2$, then the problem (2.1)-(2.4) has no solution.

For the sake of brevity we do not write down the solutions of (2.1)-(2.4) for $\alpha=1$, that will not be used in the sequel.

If $\alpha \neq-1,0,1$, then (2.1)-(2.2) still has the first integral

$$
|u+v-\lambda|^{1-\alpha}=C u v,
$$

where $C$ is a nonnegative constant, the problem (2.1)-(2.4) may be solved by quadratures and the existence and multiplicity of steady states are easily discussed (although the complete discussion is somewhat tedious because several cases must be considered, see [30]). Here we do not pursue those results any further because they are apart from the main object of this paper; some of them (i.e. those corresponding to the case $\alpha<1$ ) will be obtained in Section 3, as a by-product of global stability results. 


\section{LARGE-TIME BEHAVIOR PROPERTIES}

Here we give the main results of the paper, concerning the large time behavior of the solutions of the problem (1.1)-(1.4), (1.28), that is written again here for convenience

$$
\begin{array}{cl}
u_{t}-u_{x}=u(\lambda-u-\alpha v), & \\
v_{t}+v_{x}=v(\lambda-v-\alpha u), \quad \text { in }-1<x<1, & \text { if } t>0, \\
v=R u & \text { at } x=-1, \quad u=R v \text { at } x=1, \quad \text { if } t>0 .
\end{array}
$$

The initial conditions are

$$
u=u_{0}(x) \quad \text { and } \quad v=v_{0}(x) \quad \text { at } t=0 \text {, }
$$

where $u_{0}$ and $v_{0}$ are $C^{1}$-functions satisfying (3.3) and

$$
\begin{aligned}
& u_{0}>0, \quad v_{0}>0 \quad \text { in }-1 \leq x \leq 1, \\
& v_{0}^{\prime}+R u_{0}^{\prime}=(\alpha-1)(R-1) R u_{0}^{2} \text { at } x=-1, \\
& u_{0}^{\prime}+R v_{0}^{\prime}=(1-\alpha)(R-1) R v_{0}^{2} \quad \text { at } \mathrm{x}=1 .
\end{aligned}
$$

Global existence and uniqueness is first considered in the following theorem.

Theorem 3.1. Under the assumptions above, (3.1)-(3.7) has a unique solution in $-1 \leq x \leq 1$ and $0 \leq t<T \leq \infty$, that is a $\mathbb{C}^{1}$-function of $x$ and $t$, and satisfies

$$
\begin{aligned}
v_{x}+R u_{x}=(\alpha-1)(R-1) R u^{2} & \text { at } x=-1, \\
u_{x}+R v_{x}=(1-\alpha)(R-1) R v^{2} & \text { at } x=1, \\
u>0, \quad v>0, &
\end{aligned}
$$

in $0 \leq t<T$ and $-1 \leq x \leq 1$. Also, the interval $0 \leq t<T$ is maximal in the following sense: either (i) $T=\infty$, or (ii) $T<\infty$ and

$$
\lim \sup \left[\|u(\cdot, t)\|_{C(I)}+\|v(\cdot, t)\|_{C(I)}\right]=\infty
$$

as $t \rightarrow T^{-}$, where $I=[-1,1]$.

Proof. The semilinear problem (3.1)-(3.4) is a particular case of that considered in [31, Chap. 5 , Section 6.5]. By applying that result we get local existence and uniqueness of a $\mathfrak{C}^{1}$ solution that satisfies (3.8)-(3.9) in $x \in I, 0 \leq t \leq T_{0}$, for a certain constant $T_{0}$ that depends only on $\lambda$, $\alpha, R$ and $\left\|u_{0}\right\|_{C(I)}+\left\|v_{0}\right\|_{C(I)}$; by applying the same result, that solution is seen to be continuable to $\left[0, T_{0}\right] \cup\left[T_{0}, T_{1}\right] \cup \cdots=[0, T$, and if $T<\infty$, then (3.11) holds because otherwise the solution would be continuable beyond $t=T$. Finally, in order to prove that (3.10) holds, assume for contradiction that there is a first value of $t, t_{1}<T$, such that either $u\left(x_{1}, t_{1}\right)=0$ or $v\left(x_{1}, t_{1}\right)=0$ for a certain $x_{1} \in 1$. If $-1<x_{1}<1$ and $u\left(x_{1}, t_{1}\right)=0$ (resp., $v\left(x_{1}, t_{1}\right)=0$ ) then by integrating (3.1) (resp., (3.2)) backwards along the characteristic $x+t=x_{1}+t_{1}$ (resp., $x-t=x_{1}-t_{1}$ ), uniqueness of the resulting ODE implies that $u\left(x_{1}+t_{1}-t, t\right)=0$ (resp., $v\left(x_{1}-t_{1}+t, t\right)=0$ ) in $t_{1}-\varepsilon<t \leq t_{1}$ for a certain constant $\varepsilon>0$, and this is in contradiction with the definition of $t_{1}$. Similarly, if $x_{1}=-1$ (resp., $x_{1}=1$ ) then $u\left(x_{1}, t_{1}\right)=v\left(x_{1}, t_{1}\right)=0$ [see (3.3)], and a contradiction is reached as above, by integrating (3.1) (resp., (3.2)) backwards along the characteristic $x+t=x_{1}+t_{1}$ (resp., $x-t=x_{1}-t_{1}$ ). Thus the proof is complete. 
The following comparison result will be applied systematically below to obtain global stability results.

Lemma 3.2. Let $U$ and $V$ be $\mathfrak{e}^{1}$-functions of $x$ and $t$ in $-1 \leq x \leq 1,0 \leq t \leq T$ such that

$$
\begin{gathered}
U_{t}-U_{x}+M U \geq 0, \quad V_{t}+V_{x}+M V \geq 0 \quad \text { in }-1 \leq x \leq 1, \\
V \geq r U \text { at } x=-1, \quad U \geq r V \text { at } x=1,
\end{gathered}
$$

whenever $0 \leq t \leq T$, where $r>0$ and $M$ are given constants. If, in addition,

$$
U>0 \text { and } \quad V>0 \quad \text { in }-1 \leq x \leq 1 \text {, }
$$

at $t=0$, then (3.14) holds for all $t \in[0, T]$.

Proof. Assume for contradiction that (3.14) does not hold in $0 \leq t \leq T$. Since $U$ and $V$ are continuous and (3.14) holds at $t=0$, there is a first value of $t, t_{1}$ such that $0<t_{1} \leq T$ and either $U\left(x_{1}, t_{1}\right)=0$ or $V\left(x_{1}, t_{1}\right)=0$ for some $x_{1} \in[-1,1]$. According to (3.13) and the definition of $t_{1}$, if $V\left(-1, t_{1}\right)=0$ (resp., $U\left(1, t_{1}\right)=0$ ) then $U\left(-1, t_{1}\right)=0$ (resp., $V\left(1, t_{1}\right)=0$ ). As a consequence, we may assume that either

$$
-1 \leq x_{1}<1 \quad \text { and } \quad U\left(x_{1}, t_{1}\right)=0 \text {, }
$$

or

$$
-1<x_{1} \leq 1 \text { and } V\left(x_{1}, t_{1}\right)=0 .
$$

But if (3.15) (resp., (3.16)) holds, we may integrate the first (resp., the second) inequality (3.12) backwards along the characteristic $x+t=x_{1}+t_{1}$ (resp., $x-t=x_{1}-t_{1}$ ) and apply Gronwall's Lemma to obtain $U\left(x_{1}+t_{1}-t, t\right) \leq 0$ (resp., $V\left(x_{1}-t_{1}+t, t\right) \leq 0$ ) in $t_{1}-\varepsilon<t \leq t_{1}$ for a certain constant $\varepsilon>0$. This is in contradiction with the definition of $t_{1}$, and the result follows.

\subsection{The monotone-flow case: $\alpha \leq 0$}

If $\alpha \leq 0$ then the system (3.1)-(3.3) is cooperative and defines a monotone flow as it will be seen below. To this end we first define sub- and super-solutions of (3.1)-(3.3) as follows. A pair of $\mathrm{e}^{1}$-functions $u$ and $v$, of $[-1,1] \times[0, T]$ into $\mathbb{R}$, will be called a super-solution of (3.1)-(3.3) if they satisfy

$$
\begin{aligned}
& u_{t}-u_{x} \geq u(\lambda-u-\alpha v), \\
& v_{t}+v_{x} \geq v(\lambda-v-\alpha u), \quad \text { in }-1 \leq x \leq 1, \\
& v \geq R u \text { at } x=-1, \quad u \geq R v \quad \text { at } x=1,
\end{aligned}
$$

for all $t \in[0, T]$. Sub-solutions are defined similarly, by reversing the above inequalities.

LEMMA 3.3. If $\alpha \leq 0$, let $\left(u_{1}, v_{1}\right)$ and $\left(u_{2}, v_{2}\right)$ be a sub- and super-solution respectively of (3.1)-(3.3) in $\left[0, t_{0}\right]$. If $u_{1}>0, v_{1}>0$ in $-1 \leq x \leq 1,0 \leq t \leq t_{0}$ and

$$
u_{1}<u_{2} \quad \text { and } \quad v_{1}<v_{2} \quad \text { in }-1 \leq x \leq 1
$$

at $t=0$, then $(3.20)$ holds for all $t \in\left[0, t_{0}\right]$. 
Proof. Let $t_{1} \geq 0$ be defined by

$$
\left.t_{1}=\sup t t \in\left[0, t_{0}\right]:(3.20) \text { holds if } 0 \leq t \leq t\right] .
$$

By continuity, $t_{1}>0$. Let $K$ be a common upper bound of $u_{1}, v_{1}, u_{2}$ and $v_{2}$ in $[-1,1] \times\left[0, t_{1}\right]$ and let the constant $M$ be defined by $M=2 K-\lambda$. Then $U=u_{2}-u_{1}$ and $V=v_{2}-v_{1}$ are readily seen to satisfy the assumptions of Lemma 3.2 in $[-1,1] \times\left[0, t_{1}\right]$. As a consequence (3.20) also holds at $t=t_{1}$; by continuity $t_{1}=t_{0}$ and the result follows.

The result in Lemma 3.3 readily implies that (3.1)-(3.3) defines a monotone flow, i.e. that if $\left(u_{1}, v_{1}\right)$ and $\left(u_{2}, v_{2}\right)$ are two $\mathrm{C}^{1}$-solutions of (3.1)-(3.3) satisfying (3.20) at $t=0$, then they also satisfy (3.20) for all $t>0$.

Lemma 3.4. If $\alpha \leq 0$, let $(u, v)$ be a positive solution of (3.1)-(3.4) satisfying (3.5)-(3.7) initially and let $T>0$ be as defined in Theorem 3.1. If

$$
u_{t}>0 \quad \text { and } \quad v_{t}>0 \text { (resp., } u_{t}<0, v_{t}<0 \text { ) in }-1 \leq x \leq 1, \quad t=0,
$$

then

$$
\left.u_{t} \geq 0 \quad \text { and } \quad v_{t} \geq 0 \text { (resp., } u_{t} \leq 0, v_{t} \leq 0\right) \quad \text { in }-1 \leq x \leq 1, \quad 0<t<T .
$$

Proof. By continuity $u_{t}>0$ and $v_{t}>0$ (resp., $u_{t}<0$ and $v_{t}<0$ ) in $[-1,1] \times\left[0, t_{0}\right]$ for a certain constant $t_{0}>0$. If $0<\varepsilon \leq t_{0}$ and the functions $u_{\varepsilon}$ and $v_{\varepsilon}$ are defined as $u_{\varepsilon}(x, t)=u(x, t+\varepsilon)$ and $v_{\varepsilon}(x, t)=v(x, t+\varepsilon)$, then $\left(u_{1}, v_{1}\right)=(u, v)$ and $\left(u_{2}, v_{2}\right)=\left(u_{\varepsilon}, v_{\varepsilon}\right)$ (resp., $\left(u_{1}, v_{1}\right)=\left(u_{\varepsilon}, v_{\varepsilon}\right)$ and $\left.\left(u_{2}, v_{2}\right)=(u, v)\right)$ satisfy the assumptions in Lemma 3.3. As a consequence $u(x, t)<u(x, t+\varepsilon)$ and $v(x, t)<v(x, t+\varepsilon)$ (resp., $u(x, t)>u(x, t+\varepsilon)$ and $v(x, t)>v(x, t+\varepsilon))$ in $[-1,1] \times[0, T-\varepsilon]$ whenever $0<\varepsilon \leq t_{0}$, and the mean function theorem implies that (3.22) holds, as stated.

Theorem 3.5. If $-1<\alpha \leq 0$ and $\lambda>-(\log R) / 2$, then (3.1)-(3.3) has a unique steady state satisfying

$$
u_{s}>0 \quad \text { and } \quad v_{s}>0 \quad \text { in }-1 \leq x \leq 1 \text {, }
$$

and every solution of (3.1)-(3.7) is such that $u$ and $v$ remain uniformly bounded above for all $t>0$ and satisfy

$$
u \rightarrow u_{s} \quad \text { and } \quad v \rightarrow v_{s} \text { as } t \rightarrow \infty, \quad \text { uniformly in }-1 \leq x \leq 1 .
$$

Proof. The proof consists of 5 steps.

Step 1. There is a constant $\varepsilon_{0}$ such that if $0<\varepsilon<\varepsilon_{0}$ then the functions $u_{0}$ and $v_{0}$ defined as

$$
u_{0}(x)=v_{0}(-x)=(1-\alpha)^{-1} \sinh (\varepsilon)\left[\varepsilon-\left(\frac{\log R}{2}\right)\right] /\left[\sinh (\varepsilon)-\sinh \left(\frac{\log R}{2}\right) \mathrm{e}^{(\varepsilon-(\log R) / 2) x}\right]
$$

satisfy (3.3), (3.5)-(3.7), and the associated solution of $(3.1)-(3.7),(\bar{u}, \bar{v})$, is such that

$$
\bar{u}_{t} \geq 0, \quad \bar{v}_{t} \geq 0 \quad \text { and } \quad \int_{-1}^{1}(\bar{u}+\bar{v}) \mathrm{d} x \leq \frac{4 \lambda+2 \log R}{1+\alpha}
$$

in $-1 \leq x \leq 1,0 \leq t<T$. 
The functions $u_{0}$ and $v_{0}$ are obtained upon substitution of $\lambda$ by $\varepsilon-(\log R / 2)$ in the steady state (2.7), and multiplication by $(1-\alpha)^{-1}$. If we choose $\varepsilon>0$ sufficiently small, then $u_{0}$ and $v_{0}$ satisfy $(3.3),(3.6),(3.7)$ and

$$
\begin{aligned}
0<u_{0}, & 0<v_{0}, \\
-u_{0}^{\prime}<u_{0}\left(\lambda-u_{0}-\alpha v_{0}\right), & v_{0}^{\prime}<v_{0}\left(\lambda-v_{0}-\alpha u_{0}\right)
\end{aligned}
$$

in $-1 \leq x \leq 1$ and, according to Theorem 3.1 and Lemmas 3.3 and 3.4, the associated solution of (3.1)-(3.4), $(\bar{u}, \bar{v})$, exists in $0 \leq t<T$ and satisfies $\bar{u}>0, \bar{v}>0$ and the first two inequalities in $(3.25)$.

Now, when dividing (3.1) and (3.2) by $u$ and $v$ respectively, adding the resulting equations, integrating in $-1 \leq x \leq 1$, taking into account the boundary conditions (3.3) and replacing $(u, v)$ by $(\bar{u}, \bar{v})$, we obtain

$$
\frac{\mathrm{d}}{\mathrm{d} t} \int_{-1}^{1} \log (\bar{u} \bar{v}) \mathrm{d} x=4 \lambda+2 \log R-(1+\alpha) \int_{-1}^{1}(\bar{u}+\bar{v}) \mathrm{d} x,
$$

and, since the derivative of $\int_{-1}^{1} \log (\bar{u} \bar{v}) \mathrm{d} x$ is nonnegative (it is a monotonously increasing function in $t$ ), the third inequality in (3.25) follows and the proof of the step is complete.

Step 2. The solution of (3.1)-(3.3) considered in Step 1, $(\bar{u}, \bar{v})$, exists for all $t>0$ and satisfies (3.24), where $\left(u_{s}, v_{s}\right)$ is a steady state of (3.1)-(3.3) such that (3.23) holds.

Let us see first that there exists a constant $K \geq 1$ such that

$$
\bar{u}(x, t) \leq K \bar{u}\left(x^{\prime}, t\right)
$$

and

$$
\bar{v}\left(x^{t}, t\right) \leq K \bar{v}(x, t)
$$

for $-1 \leq x \leq x^{\prime} \leq 1$ and $\delta \leq t<T$, where $\delta=\min \{2, T / 2\}>0$. To this end assume first that $0 \leq x^{\prime}-x=\xi \leq \delta$ and integrate equation (3.1) along the characteristic line passing through the points $(x, t)$ and $(x+\xi, t-\xi)\left(=\left(x^{\prime}, t-\xi\right)\right)$ to obtain (after replacing $(u, v)$ by $\left.(\bar{u}, \bar{v})\right)$

$$
\vec{u}(x, t) / \bar{u}(x+\xi, t-\xi)=\exp \left[\int_{0}^{\xi}(-\lambda+\bar{u}(x+s, t-s)+\alpha \bar{v}(x+s, t-s)) d s\right],
$$

or, when using (3.25),

$$
\tilde{u}(x, t) / \bar{u}\left(x^{\prime}, t\right) \leq \mathrm{e}^{|\lambda| \delta+f_{0}^{f} \bar{u}(x+s, t) \mathrm{d} s} \leq \mathrm{e}^{|\lambda| \hat{\delta}+\int_{-1}^{1} \bar{\partial}(x, t) \mathrm{d} x} \leq K_{1}
$$

if $-1 \leq x \leq x^{\prime} \leq 1, x^{\prime}-x \leq \delta$ and $\delta \leq t<T$, where $K_{1}=\mathrm{e}^{|\mathrm{| \lambda |}| \hat{o}+(2 \lambda+\log R) /(1+\alpha)}$. Now, in order to cover the general case $-1 \leq x \leq x^{\prime} \leq 1\left(0 \leq x^{\prime}-x \leq 2\right)$, we can apply the last inequality successively to obtain

$$
\frac{\bar{u}(x, t)}{\bar{u}\left(x^{\prime}, t\right)} \leq K_{1}^{n}=K, \quad \text { if }-1 \leq x \leq x^{\prime} \leq 1 \quad \text { and } \quad \delta \leq t<T,
$$

where $n$ is the integer number given by $n \delta \leq 2<(n+1) \delta$; thus the inequality (3.27a) has been obtained [the second inequality (3.27b) is obtained in a completely similar way]. 
Now, when setting $x=-1$ in (3.27a) and integrating in $-1 \leq x^{\prime} \leq 1$ we obtain

$$
\bar{u}(-1, t) \leq \frac{K}{2} \int_{-1}^{1} \tilde{u}\left(x^{\prime}, t\right) \mathrm{d} x^{\prime} \quad \text { if } \delta \leq t<T .
$$

Therefore $\bar{u}(-1, t)$ is bounded [see (3.25)] and $\bar{v}(-1, t)$ is also bounded [as it follows from the boundary condition (3.3) at $x=-1$ ], and this implies [set $x=-1$ in (3.27b)] that $\bar{v}(x, t)$ is uniformly bounded in $-1 \leq x \leq 1$ and $\delta \leq t<T$. As a consequence, $\bar{u}(1, t)$ is bounded luse the boundary condition (3.3) at $x=1]$ and then $\bar{u}(x, t)$ is also uniformly bounded, as it follows by setting $x^{\prime}=1$ in (3.27a).

Once we have proved that $\bar{u}$ and $\bar{v}$ are uniformly bounded in $-1 \leq x \leq 1$ and $\delta \leq t<T$, Theorem 3.1 ensures that $\bar{u}$ and $\bar{v}$ exists for all $t>0$ (i.e. $T=\infty$ ). And, as soon as the bounds obtained above do not depend on $T$ if $T$ is sufficiently large, we can conclude that $\bar{u}$ and $\bar{v}$ are uniformly bounded for all $t>0$.

Finally, in order to prove that $(\bar{u}, \bar{v})$ satisfy $(3.24)$, notice that the monotone bounded functions $t \rightarrow \bar{u}(\cdot, t)$ and $t \rightarrow \bar{v}(\cdot, t)$ converge pointwise in $-1 \leq x \leq 1$ to some functions $u_{s}(x)$ and $v_{s}(x)$ as $t \rightarrow \infty$ and the functions $\tilde{u}$ and $\tilde{v}$, defined as $\tilde{u}(x, t)=\tilde{u}(x, t-x+1)$ and $\tilde{v}(x, t)=\bar{v}(x, t+x+1)$, also converge pointwise to $u_{s}$ and $v_{s}$ : notice that

$$
\begin{aligned}
& \bar{u}(x, t) \leq \tilde{u}(x, t) \leq \bar{u}(x, t+2) \leq u_{s}(x), \\
& \tilde{v}(x, t) \leq \tilde{v}(x, t) \leq \bar{v}(x, t+2) \leq v_{s}(x),
\end{aligned}
$$

in $-1 \leq x \leq 1, t>0$. These functions and their spatial derivatives

$$
\begin{aligned}
\tilde{u}_{x}(x, t) & =\tilde{u}_{x}(x, t-x+1)-\bar{u}_{t}(x, t-x+1) \\
& =-\bar{u}(x, t-x+1)(\lambda-\bar{u}(x, t-x+1)-\alpha \bar{v}(x, t-x+1)), \\
\tilde{v}_{x}(x, t) & =\bar{v}_{x}(x, t+x+1)+\bar{v}_{t}(x, t+x+1) \\
& =\bar{v}(x, t+x+1)(\lambda-\bar{v}(x, t+x+1)-\alpha \bar{u}(x, t+x+1)),
\end{aligned}
$$

are uniformly bounded (from above and from below), hence $\tilde{u}$ and $\tilde{v}$ are uniformly convergent, and (3.28) readily implies that $\bar{u} \rightarrow u_{s}$ and $\bar{v} \rightarrow v_{s}$ uniformly in $-1 \leq x \leq 1$ as $t \rightarrow \infty$. Finally, by using (3.29) it is readily seen that $u_{s}$ and $v_{s}$ are classical solutions of (2.1)-(2.2) [i.e. steady states of (3.1)-(3.3)], and the proof of the step is complete.

Step 3. There is a unique steady state of (3.1)-(3.3) satisfying (3.23).

Let $\left(u_{s}^{1}, v_{s}^{1}\right)$ and $\left(u_{s}^{2}, v_{s}^{2}\right)$ be two steady states of (3.1)-(3.3) satisfying (3.23), and let $\varepsilon$ be such that $0<\varepsilon<\varepsilon_{0}$ and the functions $u_{0}$ and $v_{0}$ defined in Step 1 satisfy $u_{0}<u_{s}^{k}$ and $v_{0}<v_{s}^{k}$ in $-1 \leq x \leq 1$ for $k=1$ and 2. Then, according to Lemma 3.3, the associated solution of (3.1)-(3.4) satisfies $\bar{u}<u_{s}^{k}$ and $\bar{v}<v_{s}^{k}$ in $-1 \leq x \leq 1, t \geq 0$, and the limiting steady state obtained in Step 2 is such that

$$
u_{s} \leq u_{s}^{k} \quad \text { and } \quad v_{s} \leq v_{s}^{k} \quad \text { in }-1 \leq x \leq 1 \quad \text { for } k=1 \text { and } 2 \text {. }
$$


On the other hand, the steady state satisfy (2.1)-(2.3). If (2.1) and (2.2) are divided by $u$ and $v$ respectively, the resulting equations are added and integrated in $-1 \leq x \leq 1$, the boundary conditions $(2.3)$ are taken into account and $(u, v)$ is replaced by $\left(u_{s}, v_{s}\right),\left(u_{s}^{1}, v_{s}^{1}\right)$ and $\left(u_{s}^{2}, v_{s}^{2}\right)$, then we obtain

$4 \lambda+2 \log R=(1+\alpha) \int_{-1}^{1}\left(u_{s}+v_{s}\right) \mathrm{d} x=(1+\alpha) \int_{-1}^{1}\left(u_{s}^{1}+v_{s}^{\mathrm{l}}\right) \mathrm{d} x=(1+\alpha) \int_{-1}^{1}\left(u_{s}^{2}+v_{s}^{2}\right) \mathrm{d} x$,

that, together with the inequalities above, implies $u_{s}^{1}=u_{s}^{2}=u_{s}$ and $v_{s}^{1}=v_{s}^{2}=v_{s}$, and the result follows.

Step 4 . The steady state of (3.1)-(3.3) satisfying (3.23) is such that

$$
u_{s} \rightarrow \infty \text { and } \quad v_{s} \rightarrow \infty \quad \text { uniformly in }-1 \leq x \leq 1 \text {, }
$$

as $\lambda \rightarrow \infty$ (with $\alpha$ and $R$ fixed).

Let $\left(u_{s}^{1}, v_{s}^{1}\right)$ and $\left(u_{s}^{2}, v_{s}^{2}\right)$ be the steady states of (3.1)-(3.3) corresponding to two values of $\alpha$, $\alpha_{1}$ and $\alpha_{2}$, such that $0 \geq \alpha_{1}>\alpha_{2}>-1$ (and the same values of $\lambda$ and $R$ ). According to Steps 1 and $2,\left(u_{s}^{1}, v_{s}^{1}\right)$ and $\left(u_{s}^{2}, v_{s}^{2}\right)$ may be obtained as limits from below of solutions of (3.1)-(3.3) (for $\alpha=\alpha_{1}$ and $\alpha_{2}$ respectively), that may be chosen such that (3.20) holds at $t=0$. Then, according to Lemma 3.3, (3.20) holds for all $t>0$ and we have $u_{s}^{1} \leq u_{s}^{2}$ and $v_{s}^{1} \leq v_{s}^{2}$. As a consequence, both $u_{s}$ and $v_{s}$ increase when $\alpha$ decreases and we only need to prove that (3.30) holds for $\alpha=0$; but this is readily obtained from the closed-form expressions, (2.7) or (2.8), for the steady states in that case.

Step 5. Every solution of (3.1)-(3.7) is such that (3.21) holds.

Let $(u, v)$ be any solution of (3.1)-(3.4) such that (3.5)-(3.7) holds, and let $\left(u_{1}, v_{1}\right)$ and $\left(u_{2}, v_{2}\right)$ be solutions of $(3.1)-(3.3)$ such that $\left(u_{1}(\cdot, 0), v_{1}(\cdot, 0)\right)$ is as in Step 1, with $\varepsilon>0$ sufficiently small, and $\left(u_{2}(\cdot, 0), v_{2}(\cdot, 0)\right)$ is the steady state of $(3.1)-(3.3)$ satisfying (3.23), corresponding to a sufficiently large value of $\lambda$, such that (Step 4)

$$
\begin{aligned}
u_{1}<u<u_{2}, & v_{1}<v<v_{2} \quad \text { in }-1 \leq x \leq 1, \\
u_{1 t}>0, \quad v_{1 t}>0, & u_{2 t}<0, \quad v_{2 t}<0 \quad \text { in }-1 \leq x \leq 1,
\end{aligned}
$$

at $t=0$. Then, according to Lemma 3.3, the inequalities above hold for all $t>0$ and, as in the proof of the results in Steps 1 and 2, $\left(u_{1}, v_{1}\right)$ and $\left(u_{2}, v_{2}\right)$ are seen to satisfy (3.24). Then the result in this step follows and the proof of the theorem is complete.

Remark 3.6. In the proof of Step 4 above it has been shown that the steady state of (3.1)-(3.3) satisfying (3.23), $\left(u_{s}, v_{s}\right)$, is such that both $u_{s}$ and $v_{s}$ increase as $\alpha$ decreases. The same argument shows that $u_{s}$ and $v_{s}$ increase as either $\lambda$ or $R$ increases.

THEOREM 3.7. If $-1<\alpha \leq 0$ and $\lambda \leq-(\log R) / 2$, then any solution of $(3.1)-(3.7)$ is such that $u$ and $v$ remain uniformly bounded above for all $t>0$ and satisfy

$$
u \rightarrow 0 \text { and } \quad v \rightarrow 0 \text { as } t \rightarrow \infty \text { uniformly in }-1 \leq x \leq 1 .
$$


Proof. Let $\left(u_{2}, v_{2}\right)$ be as defined in the Step 5 of the proof of Theorem 3.5. Then, it is seen that $\left(u_{2}, v_{2}\right)$ satisfies

$$
u_{2} \rightarrow u_{s} \quad \text { and } \quad v_{2} \rightarrow v_{s} \quad \text { as } t \rightarrow \infty, \quad \text { uniformly in }-1 \leq x \leq 1 \text {, }
$$

where $u_{s} \geq 0$ and $v_{s} \geq 0$ is a steady state of (3.1)-(3.3), i.e. satisfy (2.1)-(2.3). But the boundary conditions (2.3) and the uniqueness of the ODEs (2.1) and (2.2) imply that either $u_{s}=v_{s}=0$ or $u_{s}>0$ and $v_{s}>0$ in $-1 \leq x \leq 1$; the latter cannot hold because if $u_{s}$ and $v_{s}$ were strictly positive then (Step 3 of Theorem 3.5)

$$
0 \geq 4 \lambda+2 \log R=(1+\alpha) \int_{-1}^{1}\left(u_{s}+v_{s}\right) \mathrm{d} x>0 .
$$

Therefore, $(u, v)$ satisfy $(3.31)$ and the proof is complete.

We now consider the critical and sub-critical cases, $\alpha=-1$ and $\alpha<-1$ respectively.

THEOREM 3.8. A. If either $\alpha=-1$ and $\lambda>-(\log R) / 2$ or $\alpha<-1$ and $\lambda \geq-(\log R) / 2$, then every solution of (3.1)-(3.7) becomes unbounded as $t \rightarrow T \leq \infty$, where $T$ (depends on the solution and) is finite in the second case.

B. If $\alpha<-1$ and $\lambda<-(\log R) / 2$ then every solution of (3.1)-(3.7) with $\int_{-1}^{1} \log (u v) \mathrm{d} x$ sufficiently large at $t=0$ becomes unbounded in finite time.

Proof.

A. Let $(u, v)$ be a solution of (3.1)-(3.4) satisfying (3.5)-(3.7) initially. If $\alpha \leq-1$ then we may use Jensen's inequality $\left(\int_{a}^{b} \exp (f(x)) \mathrm{d} x \geq \exp \left[(b-a)^{-1} \int_{a}^{b} f(x) \mathrm{d} x\right]\right.$ if $0<b-a<\infty$, see, e.g. [32, pp. 136-138]) to obtain the following inequality from (3.26)

$$
\frac{\mathrm{d}}{\mathrm{d} t} \int_{-1}^{1} \log (u v) \mathrm{d} x \geq 4 \lambda+2 \log R-2(1+\alpha) \exp \left[\int_{-1}^{1} \log (u v) \mathrm{d} x / 4\right] .
$$

Now, from (3.32), $u$ and $v$ are readily seen to satisfy $\int_{-1}^{1} \log (u v) \mathrm{d} x \geq g(t)$, where the function $g$ is the unique solution of

$$
\begin{aligned}
g^{\prime} & =4 \lambda+2 \log R-2(1+\alpha) \exp (g / 4) \quad \text { if } t>0, \\
g(0) & =\int_{-1}^{1} \log [u(x, 0) v(x, 0)] \mathrm{d} x>0,
\end{aligned}
$$

thus, if $\alpha<-1$ and $\lambda \geq-(\log R) / 2$ we have $g(t) \rightarrow \infty$ as $t \rightarrow T<\infty$, and if $\alpha=-1$ and $\lambda>-(\log R) / 2$ then $g(t) \rightarrow \infty$ as $t \rightarrow \infty$, and the result follows.

B. As in the proof of part A, $(u, v)$ satisfy (3.32) and if

$$
\int_{-1}^{1} \log [u(0, x) v(0, x)] \mathrm{d} x>4 \log [(2 \lambda+\log R) /(1+\alpha)]
$$

then we have from (3.33) that $g(t) \rightarrow \infty$ in finite time, and the result follows. 
Remark 3.9. If $\alpha<-1, \lambda<-(\log R) / 2$ and $u_{0}$ and $v_{0}$ are sufficiently small in $-1 \leq x \leq 1$, then the solution of (3.1)-(3.4) satisfies (3.31), as it is readily proved by means of the arguments in the proof of Theorem 3.5 and in the proof of Theorem 3.7. Furthermore, if $\alpha<-1$ and $\lambda<-(\log R) / 2$, then (3.1)-(3.3) has a steady state satisfying (3.23), $\left(u_{s}, v_{s}\right)$, and $u_{s}$ and $v_{s}$ increase as $\lambda$ decreases; notice that the system (2.1)-(2.3) is invariant under the transformation $\left(u_{s}, v_{s}\right) \rightarrow\left(v_{s}, u_{s}\right) /|\alpha|, \alpha \rightarrow 1 / \alpha, \lambda \rightarrow-\lambda$ and $R \rightarrow 1 / R$ and use the results in Theorem 3.5 and Remark 3.6. Then, when using the monotony of the flow defined by (3.1)-(3.3), as in the proofs of Theorems 3.5, 3.7 and 3.8, it may be shown that if $u_{0}<u_{s}$ and $v_{0}<v_{s}$ (resp., $u_{0}>u_{s}$ and $v_{0}>v_{s}$ ) the solution of (3.1)-(3.4) satisfies (3.31) (resp., diverges in finite time).

\subsection{The nonmonotone-flow case: $\alpha \geq 0$}

If $\alpha>0$ the system (3.1)-(3.3) is no longer co-operative, the associated flow is not monotone and standard comparison methods are of limited scope. They will provide global asymptotic stability if $\lambda \leq-(\log R) / 2$ and global bounds of the solutions if $\lambda>-(\log R) / 2$. More precise global stability properties in the case $\lambda>-(\log R) / 2$ will be obtained below by means of coupled sub-and super-solutions. That technique has been successfully used to prove existence [33] and global stability properties [34-36] of parabolic systems.

We first consider the following comparison result.

LEMMA 3.10. If $\alpha>0$, let $(u, v)$ be a solution of (3.1)-(3.7), and let $(\tilde{u}, \tilde{v})$ be a solution of the problem that is obtained when $\alpha$ is set to zero in $(3.1)-(3.7)$. Then $u$ and $v$ remain uniformly bounded above for all $t>0$. If, in addition

$$
u<\tilde{u} \quad \text { and } \quad v<\tilde{v} \quad \text { in }-1 \leq x \leq 1
$$

at $t=0$, then (3.29) holds for all $t>0$.

Proof. According to Theorems 3.5 and 3.7, $\tilde{u}$ and $\tilde{v}$ are uniformly bounded above for all $t \geq 0$; then the first statement is a consequence of the second one. In order to prove the latter, notice that by continuity, if $(3.34)$ holds in $0 \leq t \leq t_{0}$ then it holds also in $t_{0} \leq t<t_{0}+\varepsilon$ for some $\varepsilon>0$. In addition (and this completes the proof), if $(3,34)$ holds in $0 \leq t<T$, then it also holds at $t=T$. In order to prove that statement, let $K$ be a common upper bound of $\tilde{u}$ and $\tilde{v}$ in $-1 \leq x \leq 1,0 \leq t \leq T$ and apply Lemma 3.2 with $U=\tilde{u}-u, V=\tilde{v}-v$ and $M=2 K-\lambda$.

THEOREM 3.11. If $\alpha>0$ then every solution of (3.1)-(3.7) is uniformly bounded above in $-1 \leq x \leq 1, t \geq 0$. If, in addition, $\lambda \leq-(\log R) / 2$, then every solution of (3.1)-(3.7) satisfies

$$
u \rightarrow 0 \quad \text { and } \quad v \rightarrow 0 \quad \text { as } t \rightarrow \infty, \quad \text { uniformly in }-1 \leq x \leq 1 .
$$

Proof. Let $\tilde{u}$ and $\tilde{v}$ be as defined in Lemma 3.10. According to Theorems 3.5 and 3.7, $\tilde{u}$ and $\tilde{v}$ are uniformly bounded above, and they converge uniformly to zero as $t \rightarrow \infty$ if $\lambda \leq-(\log R) / 2$. Then Lemma 3.10 yields the result. 
Standard comparison methods do not lead us any further. In order to obtain global stability results when $0<\alpha<1$ and $\lambda>-(\log R) / 2$ we consider coupled sub-and super-solutions of (3.1)-(3.3), $\left(u_{*}, v_{*}\right)$ and $\left(u^{*}, v^{*}\right)$, that may be defined as solutions of the following system of equations

$$
\begin{aligned}
u_{* t}-u_{* x} & =u_{*}\left(\lambda-u_{*}-\alpha v^{*}\right), \\
v_{* t}+v_{* x} & =v_{*}\left(\lambda-v_{*}-\alpha u^{*}\right), \\
u_{t}^{*}-u_{x}^{*} & =u^{*}\left(\lambda-u^{*}-\alpha v_{*}\right), \\
v_{t}^{*}+v_{x}^{*} & =v^{*}\left(\lambda-v^{*}-\alpha u_{*}\right),
\end{aligned}
$$

in $-1<x<1, t>0$, with boundary conditions

$$
\begin{array}{lll}
v_{*}=R u_{*}, & v^{*}=R u^{*} & \text { at } x=-1, \\
u_{*}=R v_{*}, & u^{*}=R v^{*} & \text { at } x=1,
\end{array}
$$

and initial conditions

$$
u_{*}=u_{0}, \quad u_{*}=u^{0}, \quad v_{*}=v_{0}, \quad v^{*}=v^{0} \quad \text { at } t=0,
$$

where $u_{0}, u^{0}, v_{0}$ and $v^{0}$ are $\mathcal{C}^{1}$-functions in $-1 \leq x \leq 1$ satisfying

$$
\begin{gathered}
0<u_{0}<u^{0}, \quad 0<v_{0}<v^{0} \quad \text { in }-1 \leq x \leq 1, \\
v_{0}=R u_{0}, \quad v^{0}=R u^{0}, \\
\mathrm{~d} v_{0} / \mathrm{d} x+R \mathrm{~d} u_{0} / \mathrm{d} x=R(1-R) u_{0}\left(u_{0}-\alpha u^{0}\right), \\
\mathrm{d} v^{0} / \mathrm{d} x+R \mathrm{~d} u^{0} / \mathrm{d} x=R(1-R) u^{0}\left(u^{0}-\alpha v_{0}\right),
\end{gathered}
$$

at $x=-1$, and

$$
\begin{gathered}
u_{0}=R v_{0}, \quad u^{0}=R v^{0}, \\
\mathrm{~d} u_{0} / \mathrm{d} x+R \mathrm{~d} v_{0} / \mathrm{d} x=R(R-1) v_{0}\left(v_{0}-\alpha v^{0}\right), \\
\mathrm{d} u^{0} / \mathrm{d} x+R \mathrm{~d} v^{0} / \mathrm{d} x=R(R-1) v^{0}\left(v^{0}-\alpha v_{0}\right)
\end{gathered}
$$

at $x=1$.

The more general definition of coupled sub- and super-solutions involve inequalities instead of equations (3.35)-(3.40), but that generality will be unnecessary in the sequel. Our results below rely on the following comparison lemma.

LEMMA 3.12. If $\alpha>0$, then:

A. The problem (3.35)-(3.48) has a unique solution in $-1 \leq x \leq 1,0 \leq t<\infty$, which is a $\mathfrak{e}^{1}$-function of $x$ and $t$, and satisfies

$$
0<u_{*}<u^{*}, \quad 0<v_{*}<v^{*} \quad \text { in }-1 \leq x \leq 1, t \geq 0 .
$$

Also, $u^{*}$ and $v^{*}$ are uniformly bounded in $-1 \leq x \leq 1,0 \leq t<\infty$.

B. Let $(u, v)$ be a solution of $(3.1)-(3.7)$ such that

$$
u_{*}<u<u^{*}, \quad v_{*}<v<v^{*} \quad \text { in }-1 \leq x \leq 1
$$

at $t=0$. Then (3.50) holds for all $t>0$. 
Proof.

A. By the argument in the proof of Theorem 3.1 we see that (3.35)-(3.48) has a unique solution in $-1 \leq x \leq 1,0 \leq t<T_{0} \leq \infty$, that is a $\mathcal{C}^{1}$-function of $x$ and $t$, and satisfies

$$
u_{*}>0, \quad v_{*}>0, \quad u^{*}>0 \text { and } v^{*}>0 \quad \text { in }-1 \leq x \leq 1 \text { and } 0 \leq t<T_{0} \text {, }
$$

and that either $T_{0}=\infty$ or

$$
\lim \sup \left[\left\|u_{*}(\cdot, t)\right\|_{C(I)}+\left\|v_{*}(\cdot, t)\right\|_{C(I)}+\left\|u^{*}(\cdot, t)\right\|_{C(I)}+\left\|v^{*}(\cdot, t)\right\|_{C(I)}\right]=\infty
$$

as $t>T_{0}$, where $I=[-1,1]$. Then the proof of this part will be complete if we show that: (i) $T_{0}=\infty$, (ii) (3.49) holds and (iii) $u^{*}$ and $v^{*}$ are uniformly bounded above in $-1 \leq x \leq 1$, $0 \leq t<\infty$. In order to prove these three statements we only need to show that if $\tilde{u}$ and $\tilde{v}$ are defined as in Lemma 3.10, then

$$
u_{*}<u^{*}<\bar{u} \text { and } v_{*}<v^{*}<\bar{v} \quad \text { in }-1 \leq x \leq 1
$$

if $0<t<T_{0}$ provided that (3.5) holds at $t=0$ (recall that $\tilde{u}$ and $\tilde{v}$ are uniformly bounded above in $-1 \leq x \leq 1,0 \leq t<\infty)$. But this statement is a consequence of the following two assertions. First, by continuity, if (3.51) holds in $0 \leq t \leq t_{0}<T_{0}$ then it also holds in $t_{0} \leq t<t_{0}+\varepsilon$ for some $\varepsilon>0$. Secondly, if (3.51) holds in $0 \leq t<T<T_{0}$, then it also holds at $t=T$; in order to prove that assertion, let $K$ be a common bound of $\tilde{u}$ and $\tilde{v}$ in $-1 \leq x \leq 1$, let the constant $M$ be defined as $M=(2+\alpha) K-\lambda$ and apply Lemma 3.2 twice, with $(U, V)=\left(\tilde{u}-u^{*}, \tilde{v}-v^{*}\right)$, and with $(U, V)=\left(u^{*}-u_{*}, v^{*}-v_{*}\right)$.

B. As above, if (3.50) holds at $t=t_{0}$, then it also holds in $t_{0}<t<t_{0}+\varepsilon$ for some $\varepsilon>0$ (continuity); and if (3.50) holds in $0 \leq t<t_{0}$ then it also holds at $t=t_{0}$ Lapply Lemma 3.2 twice, with $(U, V)=\left(u-u_{*}, v-v_{*}\right)$, and with $\left.(U, V)=\left(u^{*}-u, v^{*}-v\right)\right]$. Thus the result follows.

LEMMA 3.13. If $0<\alpha<1$ then every solution of (3.35)-(3.41) satisfies

$$
\int_{-1}^{1}\left(u^{*}-u_{*}+v^{*}-v_{*}\right) \mathrm{d} x \rightarrow 0 \quad \text { as } t \rightarrow \infty \text {. }
$$

If, in addition, $\lambda>-(\log R) / 2$, then

$$
\lim \sup \int_{-1}^{1}\left(u_{*}+v_{*}\right) \mathrm{d} x>0 \quad \text { as } t \rightarrow \infty
$$

Proof. Multiply (3.35) by $u_{*}^{-1},(3.36)$ by $v_{*}^{-1}$, add, integrate in $-1<x<1$ and take into account (3.39)-(3.40) to obtain

$$
\frac{\mathrm{d}}{\mathrm{d} t} \int_{-1}^{1} \log \left(u_{*} v_{*}\right) \mathrm{d} x=4 \lambda+2 \log R-\int_{-1}^{1}\left[u_{*}+v_{*}+\alpha\left(u^{*}+v^{*}\right)\right] \mathrm{d} x .
$$

A similar procedure applied to (3.37) and (3.38) yields

$$
\frac{\mathrm{d}}{\mathrm{d} t} \int_{-1}^{1} \log \left(u^{*} v^{*}\right) \mathrm{d} x=4 \lambda+2 \log R-\int_{-1}^{1}\left[u^{*}+v^{*}+\alpha\left(u_{*}+v_{*}\right)\right] \mathrm{d} x,
$$


and, by subtracting (3.54) from (3.55) we obtain

$$
\frac{\mathrm{d}}{\mathrm{d} t} \int_{-1}^{1} \log \left(u^{*} v^{*} / u_{*} v_{*}\right) \mathrm{d} x=-(1-\alpha) \int_{-1}^{1}\left[\left(u^{*}-u_{*}\right)+\left(v^{*}-v_{*}\right)\right] \mathrm{d} x .
$$

Now, since the right hand side of (3.56) is strictly negative (Lemma $3.12 \mathrm{~A}$ ), the function $t \rightarrow \int_{-1}^{1} \log \left(u^{*} v^{*} / u_{*} v_{*}\right) \mathrm{d} x(>0$ for all $t \geq 0)$ is strictly decreasing and bounded below; then it is convergent and

$$
\int_{0}^{\infty} \int_{-1}^{1}\left[\left(u^{*}-u_{*}\right)+\left(v^{*}-v_{*}\right)\right] \mathrm{d} x \mathrm{~d} t<\infty
$$

On the other hand, when (3.35) and (3.36) are subtracted from (3.37) and (3.38) respectively, the resulting equations are added and integrated in $-1<x<1$, and the result in Lemma 3.12A is taken into account it is seen that

$$
\left|\frac{\mathrm{d}}{\mathrm{d} t} \int_{-1}^{1}\left[\left(u^{*}-u_{*}\right)+\left(v^{*}-v_{*}\right)\right]\right|<K \quad \text { for all } t \geq 0,
$$

where $K$ is a certain constant. Since $\int_{-1}^{1}\left[\left(u^{*}-u_{*}\right)+\left(v^{*}-v_{*}\right)\right] \mathrm{d} x>0$ for all $t \geq 0$ (Lemma $3.2 \mathrm{~A})$, equations $(3.57)$ and $(3.58)$ readily imply that $(3.52)$ holds.

Finally, in order to obtain (3.53) assume for contradiction that

$$
\int_{-1}^{1}\left(u_{*}+v_{*}\right) \mathrm{d} x \rightarrow 0 \quad \text { as } t \rightarrow \infty
$$

Then from (3.52) and (3.54) we obtain

and

$$
\frac{\mathrm{d}}{\mathrm{d} t} \int_{-1}^{1} \log \left(u_{*} v_{*}\right) \mathrm{d} x \rightarrow 4 \lambda+2 \log R>0
$$

$$
\int_{-1}^{1}\left(u_{*}+v_{*}\right) \mathrm{d} x \geq \int_{-1}^{1}\left[\log u_{*}-1+\log v_{*}-1\right] \mathrm{d} x \rightarrow \infty \quad \text { as } t \rightarrow \infty .
$$

Thus the required contradiction is obtained and the proof is complete.

We now may prove the main result in this section: if $0<\alpha<1$ and $\lambda>-(\log R) / 2$ then (3.1)-(3.3) has a unique steady state that is globally asymptotically stable (with the sup norm).

THEOREM 3.14. If $0<\alpha<1$ and $\lambda>-(\log R) / 2$, then (3.1)-(3.3) has a unique steady state, $\left(u_{s}, v_{s}\right)$, such that

$$
u_{s}>0 \text { and } \quad v_{s}>0 \quad \text { in }-1 \leq x \leq 1 \text {, }
$$

and every solution of (3.1)-(3.7) satisfies

$$
u \rightarrow u_{s} \quad \text { and } \quad v \rightarrow v_{s} \quad \text { uniformly in }-1 \leq x \leq 1 \text {, as } t \rightarrow \infty \text {. }
$$


Proof. Let $(u, v)$ be any solution of (3.1)-(3.7), and let $u_{0}, v_{0}, u^{0}$ and $v^{0}$ be $\mathrm{e}^{1}$-functions of $[-1,1]$ into $\mathbb{R}$ satisfying (3.42)-(3.48) and

$$
u_{0}<u(\cdot, t)<u^{0}, \quad v_{0}<v(\cdot, t)<v^{0} \quad \text { in }-1 \leq x \leq 1,0 \leq t \leq 2 .
$$

Then the solution of $(3,35)-(3.41)$ is such that

$$
u_{*}(x, t)<u(x, t+\tau)<u^{*}(x, t), \quad v_{*}(x, t)<v(x, t+\tau)<v^{*}(x, t)
$$

if $-1 \leq x \leq 1,0 \leq t<\infty$ and $0 \leq \tau \leq 2$, as seen when applying Lemma 3.12B to the solution of (3.1)-(3.7) $(\bar{u}, \bar{v})$, where $\bar{u}(x, t) \equiv u(x, t+\tau)$ and $\bar{v}(x, t)=v(x, t+\tau)$. The proof proceeds in two steps.

Step 1 .

$$
u \rightarrow u_{s} \quad \text { and } \quad v \rightarrow v_{s} \quad \text { in } L_{1}(1-1,10 \quad \text { as } t \rightarrow \infty,
$$

where $\left(u_{s}, v_{s}\right)$ is a steady state of (3.1)-(3.3) satisfying (3.59).

Let us consider the functions $U$ and $V$ defined as

$$
U(x, t)=\int_{t}^{t+1} u(x, \sigma) \mathrm{d} \sigma, \quad V(x, t)=\int_{t}^{t+1} v(x, \sigma) \mathrm{d} \sigma,
$$

that satisfy [see (3.60)]

$$
u_{*}<U<u^{*} \quad \text { and } \quad v_{*}<V<v^{*} \quad \text { in }-1 \leq x \leq 1,0 \leq t<\infty .
$$

Then $|U-u|$ and $|V-v|$ are bounded by $u^{*}-u_{*}$ and $v^{*}-v_{*}$ in $-1 \leq x \leq 1,0 \leq t<\infty$. Since, in addition, $\left|U_{t}(x, t)\right|=|u(x, t+1)-u(x, t)|$ and $\left|V_{t}(x, t)\right|=|v(x, t+1)-v(x, t)|$ are also bounded by $\left(u^{*}-u_{*}\right)$ and $\left(v^{*}-v_{*}\right)$ respectively, we have

$$
\begin{aligned}
U-u & \rightarrow 0, & V-v & \rightarrow 0, \\
U_{t} & \rightarrow 0, & V_{t} & \rightarrow 0,
\end{aligned}
$$

in $L_{1}(]-1,1$ [) as $t \rightarrow \infty$, as obtained by applying Lemma 3.13. Also [see (3.57)]

$$
\int_{0}^{\infty} \int_{-1}^{1}\left(\left|U_{t}(x, t)\right|+\left|V_{t}(x, t)\right|\right) \mathrm{d} x \mathrm{~d} t<\infty,
$$

and this implies that

$$
\int_{-1}^{1}(|U(x, t+\sigma)-U(x, t)|+|V(x, t+\sigma)-V(x, t)|) \mathrm{d} x \rightarrow 0
$$

uniformly in $0 \leq \sigma<\infty$, as $t \rightarrow \infty$; as a consequence

$$
\left.\left.U \rightarrow u_{s} \quad \text { and } \quad V \rightarrow v_{s} \quad \text { in } L_{1}(]-1,1\right]\right) \quad t \rightarrow \infty .
$$

Also, it follows from (3.63) and (3.65) that $u \rightarrow u_{s}$ and $v \rightarrow v_{s}$ in $L_{1}(]-1,1[)$ as $t \rightarrow \infty$, and the same is true for $u^{*}, u_{*}$ and $v^{*}, v_{*}$ respectively (see Lemma 3.13), and using the functions $\hat{u}$ and $\tilde{v}$, defined as

$$
\tilde{u}(x, t)=u(x, t-x+1) \quad \text { and } \quad \tilde{v}(x, t)=v(x, t+x+1),
$$


if $-1 \leq x \leq 1$ and $t>0$, it is readily seen from (3.60) that $\bar{u} \rightarrow u_{s}$ and $\tilde{v} \rightarrow v_{s}$ in $\left.L_{1}(]-1,1\right]$ as $t \rightarrow \infty$. Apply now the argument at the end of Step 2 of the proof of Theorem 3.5 to obtain that $\tilde{u} \rightarrow u_{s}$ and $\tilde{v} \rightarrow v_{s}$ uniformly in $-1 \leq x \leq 1$ as $t \rightarrow \infty ;\left(u_{s}, v_{s}\right)$ is a classical solution of (2.1)-(2.3). Finally, when taking into account (3.66) we can conclude that $u \rightarrow u_{s}$ and $v \rightarrow v_{s}$ uniformly in $-1 \leq x \leq 1$ as $t \rightarrow \infty$, and the proof of the step is complete.

Step 2. (3.1)-(3.3) possess at most one steady state satisfying (3.59).

Assume for contradiction that two such steady states, $\left(u_{s}^{1}, v_{s}^{1}\right)$ and $\left(u_{s}^{2}, v_{s}^{2}\right)$, exist. If the initial conditions for (3.35)-(3.40) are taken such that

$$
u_{0}<u_{s}^{k}<u^{0} \quad \text { and } \quad v_{0}<v_{s}^{k}<v^{0} \quad \text { in }-1 \leq x \leq 1, \quad \text { for } k=1 \text { and } 2 \text {, }
$$

then, according to Lemma $3.12 \mathrm{~A}$, we have

$$
u_{*}<u_{s}^{k}<u^{*} \quad \text { and } \quad v_{*}<v_{s}^{k}<v^{*} \quad \text { in }-1 \leq x \leq 1,0 \leq t<\infty
$$

for $k=1$ and 2, and (3.52) implies that

$$
\int_{-1}^{1}\left(\left|u_{s}^{1}-u_{s}^{2}\right|+\left|v_{s}^{1}-v_{s}^{2}\right|\right) \mathrm{d} x=0 .
$$

Then $u_{s}^{1} \equiv u_{s}^{2}$ and $v_{s}^{1}=v_{s}^{2}$ the required contradiction is obtained and the step and the proof of the theorem are complete.

REMARK 3.15. Numerical results [30] suggest that the conclusion in Theorem 3.14 remains true if either (i) $\alpha=1$ and $\lambda>-(\log R) / 2$ or (ii) $\alpha>1$ and $-(\log R) / 2<\lambda<\lambda_{C}$ for some constant $\lambda_{c}$. Nevertheless, this result cannot be obtained with the ideas in this section because, if $\alpha \geq 1$ then the quantity

$$
\int_{-1}^{1} \log \left(u^{*} v^{*} / u_{*} v_{*}\right) \mathrm{d} x
$$

does not converge to zero as $t \rightarrow \infty$ [see (3.56)].

\section{CONCLUDING REMARKS}

We have proven the results described at the end of Section 1. Three remarks concerning these results and their extensions are in order:

(a) If the initial conditions $u_{0}$ and $v_{0}$ are allowed to vanish, then the dynamics of (1.1)-(1.3) become quite complex [30]. That complexity is due to the fact that the number of zeroes of $u$ and $v$ remains constant as $t$ increases (the zeroes propagate along the characteristics), and is nongeneric (see [30]) and spurious because the second spatial derivatives in (1.12)-(1.13), that have been neglected, do not allow zeroes of $|A|^{2}$ and $|B|^{2}$ to remain as time proceeds.

(b) Notice that we have paid only small attention to the case $\alpha=-1$ [we only obtained the results (iv) and (v) at the end of Section 1, whose proof was very simple]. The reason is that equations (3.1)-(3.3) are not a good approximation of the amplitude equations (1.12)-(1.13) in this critical case, when higher order nonlinear (quintic,...) terms cannot be neglected.

(c) If $\alpha \geq 1$ and $\lambda>-(\log R) / 2$ then our only conclusion is that in (vii), and the ideas in this paper do not allow to obtain further results concerning the asymptotic behavior as $t \rightarrow \infty$ (Remark 3.15). An analysis of the dynamics of (1.1)-(1.7) in that case is presented in [30], by 
means of continuation methods and typical dynamical systems techniques. Those results show that the conclusion (vi) is no longer true if $\alpha>1$, when for appropriate values of $\lambda,(1.1)-(1.7)$ exhibits quite complex dynamics as $t \rightarrow \infty$; including periodic and quasi-periodic behaviors, intermittency, period-doubling sequences and crises (i.e. chaotic collisions of nonchaotic attractors) associated with symmetry gaining from nonsymmetric limit cycles (the underlying symmetry being given by the map $A \leftrightarrow B$ and $x \rightarrow-x$ ).

\section{REFERENCES}

I. CROSS M. C. \& HOHENBerg P. C., Pattern formation outside of equilibrium, Rev. Modern Phys. 65, 851-1112 (1993).

2. STEINBERG V., FINEBERG J., MOSES E. \& REHBERG 1., Pattern selection and transition to turbulence in propagating waves, Physica $D$ 37, 359-383 (1989).

3. BENSIMON D., KOLODNER P., SURKO C. M., WILLIAMS H. \& CROQUETTE V., Competing and coexisting dynamical states of travelling-wave convection in an annulus, J. Fluid Mech. 217, 441-467 (1990).

4. KOLODNER P., Repeated transients of weakly nonlinear travelling-wave convection, Phys. Rev. E 47, 1038-1048 (1993).

5. KAPLAN E. \& STEINBERG V., Measurement of reflection of travelling waves near the onset of binary-fluid convection, Phys. Rev. E 48, R661-R664 (1993).

6. PREDTECHENSKY A. A., McCORMICK W. D., SWIFT J. B., NOSZTICZIUS Z. \& SWINNEY H. L., Onset of travelling waves in isothermal double diffusive convection, Phys. Rev. Lett. 72, 218-221 (1994).

7. MOTSAY R. W., ANDERSON K. E. \& BEHRINGER R. P., The onset of convection and turbulence in rectangular layers of normal liquid ${ }^{4} \mathrm{He}$, J. Fluid Mech. 189, 263-286 (1988).

8. CROQUETTE V. \& WILLIAMS H., Nonlinear competition between waves on convective rolls, Phys. Rev. A 39, 2765-2768 (1989).

9. CROQUETTE V. \& WILLIAMS H., Nonlinear waves of the oscillatory instability on finite convective rolls, Physica D 37, 300-314 (1989).

10. ANDERECK C. D., LIU S. S. \& SWINNEY H. L., Flow regimes in a circular Couette system with independently rotating cylinders, J. Fluid Mech, 164, 155-183 (1986).

11. LANGFORD W. F., TAGG R., KOSTELICH E. J., SWINNEY H. L. \& GOLUBISTKY M., Primary instabilities and bicriticality in flow between counter-rotating cylinders, Physics Fiuids 31, 776-785 (1988).

12. DUBOIS M., DAVIAUD F., RONSIN O. \& BERGE P., Traveling waves in pure fluids heated along wires, Physica D 61, 140-146 (1992).

13. VINCE J. M. \& DUBOIS M., Hot wire below the free surface of a liquid: structural and dynamical properties of a secondary instability, Europhys. Lett. 20, 505-510 (1992).

14. DAVIAUD F. \& VINCE J. M., Traveling waves in a fluid layer subjected to a horizontal temperature gradient, Phys. Rev. E 48, 4432-4436 (1993).

15. JOETS A. \& RIBOTTA R., Localized, time-dependent state in the convection of a nematic liquid crystal, Phys. Rev. Lett. 60, 2164-2167 (1988).

16. REHBERG 1., RASENAT S. \& STEINBERG V., Traveling waves and defect-initiated turbulence in electroconvecting nematics, Phys, Rev, Lett. 62, 756-759 (1989).

17. REHBERG I., WINKLER B. L., DE LA TORRE M., RASENAT S. \& SCHÖPF W., Pattern formation in a liquid crystal, Advances in Solid State Physics 29 (Ed. V. ROESSLER), pp. 35-52. Vieweg, Braunschweig (1989).

18. DE LA TORRE M., Contribución al estudio de la formación de estructuras en fluidos anisótropos. Doctoral Thesis, Universidad Nacional de Educación a Distancia, Madrid (1990).

19. MARKSTEIN G. H., Nonsteady Flame Propagation. Plenum Press (1964).

20. FERGUSON C. R. \& KECK J. C., Stand-off distances on a flat burner, Combustion and Flame 34, 85-98 (1979).

21. SABATHIER F., BOYER L. \& CLAVIN P., Experimental study of weak turbulent premixed flames, Combustion and Flame 35, 139-153 (1979).

22. COULLET P., FAUVE S. \& TIRAPEGUI E., Large scale instability of nonlinear standing waves, $J$. Physique Lett. 46, L787-L791 (1985).

23. CROSS M. C., Travelling and standing waves in binary-fluid convection in finite geometries, Phys. Rev. Lett. 57, 2935-2938 (1986).

24. MARTEL C., La inestabilidad oscilatoria y sus aplicaciones en Mecánica de Fluidos y Combustión. Doctoral Thesis, Universidad Politéenica de Madrid, Madrid (1995).

25. MARTEL C. \& VEGA J. M., Finite size effects near the onset of the oscillatory instability, Nonlinearity 9, 1129-J17I (1996).

26. CROSS M. C. \& KUO E. Y., One dimensional spatial structure near a Hopf bifurcation at finite wavenumber, Physica D 59, 90-120 (1992). 
27. KNOBLOCH E. \& DE LUCA J., Amplitude equations for traveling wave convection, Nonlinearity 3, 575-580 (1990).

28. ALVAREZ-PEREIRA C. \& VEGA J. M., On the pulsating instability of two-dimensional flames, Eur. J. Appl. Math. 3, 55-73 (1992).

29. VEGA J. M., On the amplitude equations arising at the onset of the oscillatory instability in pattern formation, SIAM J. math. Analysis 24, 603-617 (1993).

30. MARTEL C. \& VEGA J. M., Dynamics of a hyperbolic system that applies at the onset of the oscillatory instability, Noninearity (submitted).

31. COURANT R. \& HILBERT D., Methods of Mathematical Physics, Vol. II. Interscience (1962).

32. HARDY G. H., LITTLEWOOD J. E. \& POLYA G., Inequalities. Cambridge University Press (1967).

33. LADDE G. S., LAKSHMIKANTHAM V. \& VATSALA A. S., Existence and asymptotic behavior of reactiondiffusion systems via coupled quasi-solutions, Nonlinear Analysis and Applications (Ed. V. LAKSHMIKANTHAM). Academic Press (1982).

34. LEUNG A. \& CLARK D., Bifurcations and large time behavior for prey-predator reaction-diffusion equations with Dirichlet boundary data, $J$. diff. Eqns 35, 113-127 (1980).

35. HERNÁNDEZ J., Some existence and stability results for solutions of reaction-diffusion systems with nonlinear boundary conditions, Nonlinear Differential Equations: Invariance, Stability and Bifurcation (Ed. P. DE MOTTON1). Academic Press (1981).

36. VEGA J. M., Invariant regions and global asymptotic stability in an isothermal catalyst, $S I A M J$. math. Analysis 19. 774-796 (1988). 